\title{
LER: objeto e pretexto para a construção do campo trabalho e saúde
}

\author{
RSI: object and pretext for constructing \\ the field of Workers' Health
}

Leny Sato 1

\footnotetext{
1 Departamento de Psicologia Social e do Trabalho, Instituto de Psicologia, Universidade de São Paulo. Av. Prof. Mello Moraes 1721, São Paulo, $S P$ 05508-900, Brasil. lenysato@usp.br
}

\begin{abstract}
This article presents a proposal for a research and action agenda in Workers' Health, taking the case of repetitive strain injury (RSI). It assumes that RSIs are both an object and a pretext for the Workers' Health area. As an object, RSIs demand both an understanding and resolution of problems as they present, and as a pretext, RSIs underscore the limits and contradictions of the paradigms adopted in Workers' Health. The article draws on data and questions raised in several studies.

Key words Repetition Strain Injury; Occupational Health; Occupational Diseases

Resumo O presente artigo tem por objetivo propor uma agenda de pesquisa e de atuação em saúde do trabalhador, tomando-se o caso das Lesões por Esforços Repetitivos (LER). Assume-se que as LER são simultaneamente objeto e pretexto para o campo da saúde do trabalhador. Como objeto, as LER demandam o equacionamento e a resolução de problemas e, como pretexto, elas apontam para os limites e para as contradições das práticas e dos paradigmas adotados em saúde do trabalhador. Para tanto, recorre a achados e questões já identificados em alguns estudos.

Palavras-chave Traumatismo por Distensão Repetida; Saúde Ocupacional; Doenças Ocupacionais
\end{abstract}




\section{Introdução}

O crescimento das Lesões por Esforços Repetitivos (LER) tem obrigado - quer pela freqüência com que têm sido diagnosticadas, quer pela ausência de preconceitos em relação a categorias profissionais as quais atinge - trabalhadores e empresários, órgãos governamentais das áreas da saúde, do trabalho e da previdência social, universidades, cada um a seu modo, a lidar com esse problema. Ademais, a importância de sua manifestação faz com que as LER sejam consideradas um grave problema de saúde pública. Esses certamente são motivos fundamentais que têm conduzido a essa obrigatoriedade. Além de sua importância quantitativa, as LER canalizam diversos aspectos que não tiveram, entre nós, outros pretextos tão fortes para tanto, requerendo respostas e suscitado diversas questões.

Assim é que, se de um lado não se pode negar que as LER se constituem em um objeto sobre o qual o campo trabalho e saúde deva se debruçar, de outro elas são um pretexto para que esse mesmo campo se construa e se desenvolva no Brasil. É nessa dupla vertente que destacaremos algumas das dimensões que as fazem ter essa dupla fecundidade, e, assim, optamos por organizar esse artigo pontuando os diversos temas, problemas e objetos de pesquisa postos pelas lesões por esforços repetitivos. Nossa pretensão, contudo, mantém-se longe de esgotar a discussão sobre cada um dos tópicos a seguir apresentados, restringindo-se a apontar para uma agenda de pesquisa e de atuação da sociedade civil e dos órgãos governamentais.

Para construir essa agenda, baseamo-nos em alguns estudos sobre a temática, como se segue:

- Diferentemente das doenças profissionais, as LER não respeitam as fronteiras entre as categorias profissionais, o que leva a se questionarem as entidades de representação dos trabalhadores sobre a sua política em saúde do trabalhador, inquirindo-as especialmente no que se refere à manutenção ou superação de estratégias corporativas, mais ainda quando a prática da terceirização tal qual a adotada pelas empresas no Brasil conforma contextos de trabalho que oferecem maior risco aos trabalhadores terceirizados de desenvolver tais lesões (Blanco, 1994). Nesse fim de século, observamos mais claramente as relações de interdependência entre os mercados formal e informal, entre as empresas "limpas" e as empresas "sujas". Segundo Salerno (1999), as primeiras seriam caracterizadas pelos altos investimen- tos em automação, mudanças nas formas de organização do processo de trabalho, grupos denominados semi-autônomos, redução de níveis hierárquicos, ampliação do espaço de autonomia para a tomada de decisões. Já as empresas "sujas" seriam caracterizadas por exigirem o cumprimento de longas jornadas, de horários irregulares de trabalho, apresentarem condições penosas e perigosas. Na visão de Salerno (1999), as grandes empresas "jogam para fora" o trabalho sujo, mas dele dependem. O mesmo é evidenciado por Navarro (1999), que aponta, ainda, a utilização do trabalho domiciliar, realizado principalmente por mulheres e crianças, para completar o processo produtivo, tomando o caso da produção de calçados em Franca, São Paulo.

- As LER exemplificam-nos uma estratégia de prática sindical para interferir na adoção de políticas públicas na área de saúde do trabalhador, mostrada por Rocha (1989) ao acompanhar a trajetória construída pelos trabalhadores desde as primeiras atuações de cipeiros (no Banco do Brasil, no início da década de 80, em Porto Alegre, até um movimento que abarca entidades sindicais de outros estados para o reconhecimento, pela Previdência Social, em 1987, de uma "nova" doença. Posteriormente, essa articulação intersindical motivou a alteração da Norma Regulamentadora 17, presente na legislação de Segurança e Medicina do Trabalho da Consolidação das Leis do Trabalho, em 1990 (Brasil, 1997); em seguida, deu-se a elaboração da norma técnica da Secretaria de Estado da Saúde, em 1992 (São Paulo, 1992), e, por fim, a da Previdência Social em 1993 (MPS, 1993).

- Às práticas sindicais acima referidas relaciona-se um outro aspecto também bastante importante, o qual diz respeito às relações entre a presença da lesão e a consciência dos trabalhadores sobre sua condição e posição no processo de produção, bem como sobre a necessidade de atuação política. Isso ocorre porque a presença da lesão constitui-se em um momento de ruptura que exige dos trabalhadores por ela acometidos a reflexão sobre sua nova condição, o que se faz rememorando e reavaliando a estória passada e projetando a ação futura. Esse, por exemplo, é o trabalho de Duarte (1998), o qual, com base em estudo de caso de um trabalhador acometido pela LER, acompanha o movimento de sua consciência. Conclui o autor que a consciência desse trabalhador sofre um processo de transformação ao encontrar interlocutores que o reconheçam como pessoa e dêem espaço para refletir sobre sua condição de trabalho e de vida, dando novo significado à sua estória. Por sua vez, Lima 
(1997) focaliza o processo de construção social da militância de trabalhadores com LER, através do caso de uma associação de portadores das lesões. Em ambos os estudos, está presente a constatação de que a vivência como portador de LER e a interação em ambientes sociais confirmadores dessa percepção são situações facilitadoras para a emergência de sujeitos com a potencialidade para transformar os contextos de vida e os scripts socialmente definidos. São essas relações interpessoais e sociais compreensivas e solidárias que possibilitam criar um substrato para práticas coletivas e individuais que questionem os papéis sociais.

- Essas lesões demandam a criação de práticas nos serviços públicos de saúde por parte de diversos profissionais de saúde, como médicos, engenheiros, psicólogos, terapeutas ocupacionais, fisioterapeutas, ergonomistas, assistentes sociais, dentre outros, obrigando-os a criar uma linguagem de interface para trabalharem com as LER, sem que se percam as especificidades de suas formações de origem. Nesse sentido, as LER podem estar sendo um objeto privilegiado para que, de fato, crie-se um campo de práticas interdisciplinares em saúde e trabalho.

- A peculiaridade de sua manifestação física - diagnóstico clínico, fortemente baseado na percepção do trabalhador sobre o próprio corpo e sobre os seus sentimentos - leva-nos a refletir sobre a relação sujeito-objeto na prática médica, questionando aquela que tem sido bastante disseminada contemporaneamente, qual seja, a de basear a atribuição diagnóstica preferencialmente em exames subsidiários, preterindo a fala do trabalhador. Fortalecendo a visão do senso comum sobre os processos de adoecimento, Bernardino (1998) descreve as representações sociais que os trabalhadores acometidos pelas LER criam sobre estas, focalizando o que é, como se manifesta e quais as repercussões dessas lesões para a vida das pessoas. Por outro lado, as LER questionam o trabalhador sobre o quanto percebe e valoriza a percepção que tem do próprio corpo e, em virtude disso, o quanto pode ser sujeito na relação médico-paciente. Essa é uma importante dimensão, pois sabemos, como já nos mostrou Boltanski (1984), que a percepção do corpo é fortemente influenciada pela posição social de classe e, como mostrou Dejours (1980), que a ideologia da vergonha presta-se a eufemizar a percepção das dores, dos males e a dilatar o limite subjetivo. Lima \& Oliveira (1995) mostram como a prática social de desconfirmação (Watzlawick et al., 1993) da percepção da doença, vivenciada pelos acometidos pela LER em vários âmbitos de relacionamentos sociais (família, colegas e superiores e profissionais de saúde), reforçam esse processo de eufemização da percepção.

- Ainda decorrente da peculiaridade da manifestação das LER - são potencialmente doenças incapacitantes, os métodos de cura e tratamento aqui adotados são múltiplos e informados por orientações filosóficas diversas e têm repercussões para a vida de trabalho e social -, existe um campo fértil que tem demandado a oferta de práticas nos serviços de saúde, referente ao sofrimento psíquico que os adoecidos pelas LER expressam. Essa demanda fez com que profissionais da área de psicologia e psiquiatria e, posteriormente, de outras especialidades, propusessem uma modalidade de assistência psicossocial a esses trabalhadores. Tal tipo de assistência visa oferecer um espaço para a "re-significação" da situação de vida, focalizada na realidade desses trabalhadores e objetivando melhorar a qualidade de vida apesar da doença (Sato, 1992a; Sato et al., 1993; Lima \& Oliveira, 1995; Araújo, 1999). Aguiar (1998) corrobora a necessidade de oferta de assistência psicossocial ao estudar trabalhadores acometidos pelas LER que estão afastados do trabalho e mostra as repercussões psicossociais da situação de afastamento, adotando como eixo a identidade. Em seus dizeres, os trabalhadores são "içados de seu cotidiano” (Aguiar, 1998), representando uma ruptura no projeto de vida até então elaborado e demandando espaço para que ele seja reconstruído. Dadas as repercussões psicossociais negativas que a situação de afastamento do trabalho provoca, questiona-se a possibilidade de adoção de outras práticas a fim de evitar que se agrave a lesão e que o sofrimento emocional seja agudizado.

- Essa forte associação entre LER e sofrimento psíquico - como mostram os estudos de Ribeiro (1995), Rego \& Santana (1995) e Borges (1999) -, além de demandar uma atenção específica na prática dos serviços, introduz questionamentos em relação ao tipo e natureza dessa associação que merecem ser investigados. E, nesse sentido, algumas hipóteses têm sido levantadas: seria a população ocupacionalmente exposta ao risco de LER a mesma exposta a riscos à saúde mental? seria possível determinar o tipo de relação que se estabelece entre elas causalidade, conseqüência, conjunção? Lucire (1986), por exemplo, explica a manifestação das LER como conversão histérica, porém existem críticas metodológicas, conceituais, ao caráter ideológico dessa concepção (Hopkins \& Russell, 1988, apud Lima \& Oliveira, 1995; Sato et al., 1993). Borges (1999) também evidencia, 
em estudo com a categoria bancária, o equívoco em explicar as LER como conversão histérica, ao demonstrar que a prevalência de distúrbios mentais menores está associada com o tipo de inserção dos trabalhadores no processo de trabalho bancário e não a outras variáveis diferentes de trabalho (características pessoais e gênero, por exemplo).

- A presença simultânea das LER e do sofrimento emocional tem sido um substrato fértil para fazer com que se expressem concepções sobre o que é o psicológico, bem como sobre a causalidade das lesões. O psicológico tem recebido conotações morais fortes por parte de profissionais de saúde vinculados a convênios médicos, empresas e INSS; de trabalhadores pertencentes a níveis hierárquicos diversos das empresas em que trabalham; de familiares dos acometidos pelas LER e, inclusive, destes próprios. Essa conotação, na qual se ressalta a visão de que o trabalhador é fraco e frágil, de que está relacionado com vontade e preguiça, é uma construção simbólica propícia para gerar e sustentar sentimentos de culpa em relação à doença; culpa que concentra a busca da explicação de sua causa no jeito de ser de cada um e não no contexto de trabalho (Sato, 1992b; Sato et al., 1993; Lima \& Oliveira, 1995). Ao focalizar no indivíduo a causa final, simultaneamente opera-se o processo de culpabilização do trabalhador pela doença.

- Uma outra relação que se mostra cada vez mais importante a ser investigada é entre gênero, trabalho e LER, pois é na população de mulheres que se tem diagnosticado a maioria dos casos de LER. Além disso, sabe-se que há diferenças de prevalência de suspeição de casos de sofrimento psíquico entre a população feminina e masculina. Estudos sobre gênero e trabalho mostram haver uma sexualização do posto de trabalho (Rodrigues, 1992) com menor grau de controle sobre a tarefa naquelas funções ditas femininas (Hall, 1989) e forte presença dos processos de socialização que geram a qualificação feminina para o mercado de trabalho (Hirata, 1988) ou, como refere Rodrigues (1978), a modalidade feminina do habitus. Ao se evidenciar a construção social das diferenças sexuais, ou seja, da questão de gênero (Spink, 1994), torna-se explícita, no caso das LER, a construção social dos padrões de adoecimento mediada pela composição de populações de risco. Essa perspectiva demonstra a importância em incorporar categorias antropológicas, sociológicas e psicossociais para aprofundar o entendimento das LER.

- A prevenção das LER é um outro aspecto bastante importante e, em virtude da sua cau- salidade, já extensamente demonstrada por Chatterjee (1987), Thouvenin (1990), Kurppa (1979), Armstrong (1987), dentre outros, não se tem dúvida de que é a organização do trabalho que deve ser modificada, especialmente quanto ao grau de controle do trabalhador sobre a tarefa, ou seja, deve-se modificar a relação trabalhador-trabalho e não diminuir-se a exposição a fatores de risco. Ao se pensar em estratégias de prevenção, impõe-se, nesse caso, a negociação, entre patrões e empregados, de outras formas de se trabalhar, pois entendemos que não há um desenho mais adequado que venha a substituir os hoje existentes e, nessa perspectiva, consideramos que a teoria da Escola Sociotécnica contém os princípios que norteiam essa prática (Trist, 1976; Spink, 1992; P. Spink, comunicação pessoal). Porém, levando-se em conta que as condições de negociação são socialmente dadas, deve-se identificálas e/ou criá-las. Observamos que há, cotidianamente, processos de micronegociações no "chão de fábrica", os quais visam contemplar os interesses de diversas ordens, inclusive, os dos trabalhadores (Sato, 1997). Ao lado disso, a educação em saúde é uma outra prática que precisa ser estimulada junto às populações de risco, sendo também uma das propostas que os próprios portadores de LER, nos grupos de qualidade de vida, espontaneamente apresentam. Nessas atividades, parece-nos importante focalizar, dentre outros aspectos, a importância de se reconhecer e respeitar o limite subjetivo. - A reabilitação profissional e reinserção dos portadores de LER no mercado de trabalho é um outro aspecto enfrentado atualmente, que tem mostrado, segundo Settimi et al. (1995), as dificuldades que marcam essas tentativas. Qualificação, presença de sintomas da doença, carteira de trabalho "suja" (por constar registro de doença do trabalho), dentre outros, são motivos que se associam ao universo de trabalho e de emprego visível a esses trabalhadores e às ofertas de emprego, dificultando essa reinserção e a reabilitação.

- As lesões por esforços repetitivos configuram-se como uma doença que questiona paradigmas e práticas na área de saúde do trabalhador, mas, ao mesmo tempo, apresenta-se como uma peça arqueológica, pois, como demonstra Ribeiro (1997), a evolução histórica das lesões ósteo-articulares, já referidas por Ramazzini no século XVIII, pode ser entendida à luz das sucessivas modificações do trabalho, do processo de trabalho e das relações de trabalho no decorrer da nossa história. Mostra Ribeiro (1997) que as condições e a organização do trabalho, bem como a base técnica empre- 
gada, hoje sustentada na informatização e automação, foram modificadas e, com elas, as diferenças de demanda de esforço e o crescimento das LER. No momento atual, tais modificações conduziram a uma nova posição do trabalho vivo enquanto criador de valor, o que tem levado autores como Offe (1989) e Gorz (1987) a questionarem a centralidade da categoria trabalho. Em virtude disso, as LER têm o poder de remeter-nos mais rapidamente à reflexão sobre a história, a ideologia, e às relações de trabalho.

Esses são alguns dos aspectos que emergem em relação às LER, os quais requerem respostas e suscitam novas questões para os diferentes segmentos da sociedade e para instituições diversas. Certamente, não são apenas as lesões por esforços repetitivos que têm requerido respostas múltiplas e originais, apontando as limitações que os aportes teóricos clássicos conservam e instigando-nos a buscar outros, mas entendemos que essas lesões têm tido a capacidade de canalizar muitas respostas.

Diante do exposto, esperamos ter demonstrado por que optamos por manter a denominação LER nesse artigo, uma vez que, para muito além de um nome que designa uma determinada patologia, ela expressa uma série de significados construídos na dinamização de

\section{Referências}

AGUIAR, M. C. F., 1998. O Trabalhador sem seu Trabalho: Um Estudo sobre a Identidade de Trabalhadores Afastados do Trabalho por Adoecimento Profissional. Dissertação de Mestrado, Vitória: Universidade Federal do Espírito Santo.

ARAÚJO, M. D., 1999. O que é ser bancário? Categoria em extinção? In: Psicologia: Questões Contemporâneas (M. E. B. Barros, org.), pp. 199-209, Vitória: Editora da Universidade Federal do Espírito Santo.

ARMSTRONG, T. J.; FINE, L. J.; GOLDSTEIN, S. A.; LIFSHITZ, Y. R. \& SILVERSTEIN, B. A., 1987. Ergonomics considerations in hand and wrist tendinitis. Journal of Hand Surgery, 12:830-837.

BERNARDINO, M. T. S. M., 1998. Lesões por Esforços Repetitivos - LER. A Doença para o Indivíduo. Dissertação de Mestrado, São Paulo: Faculdade de Saúde Pública, Universidade de São Paulo.

BLANCO, M. C., 1994. O processo de terceirização nos bancos. In: Terceirização - Diversidade e Negociação no Mundo do Trabalho (H. S. Martins \& J. R. Ramalho, org.), pp. 76-84, São Paulo: Editora Hucitec/Núcleo de Estudos sobre Trabalho e Sociedade, Centro Ecumênico de Documentação e Informação. processos sociais, mediante diversos desafios e questionamentos: do conhecimento acumulado e da prática informada pelas diversas áreas do conhecimento; das práticas costumeiras por parte das gerências das empresas, dos trabalhadores, do movimento sindical, dos profissionais de saúde e da academia. Por esse motivo, entendemos serem as LER um típico caso que exemplifica o processo de construção da área de saúde do trabalhador, tal qual evidenciado por Lacaz (1997) e Minayo-Gomez \& Thedim-Costa (1997). É uma área que se constitui como um campo teórico e de práticas, conformado pela interação de diversos atores sociais e instituições, estabelece diálogo cooperativo e conflituoso entre as diversas disciplinas do conhecimento e funda-se no bojo da medicina social latino-americana. Vale ressaltar que essa dinâmica conforma a política social em saúde voltada aos trabalhadores.

As LER constituem um objeto para a saúde do trabalhador, já que demandam o equacionamento e a resolução dos problemas apresentados, mas também são um pretexto para o avanço da construção desse campo de conhecimento, pois aponta limites e contradições das práticas e dos paradigmas que as norteiam.

BOLTANSKI, L., 1984. As Classes Sociais e o Corpo. Rio de Janeiro: Edições Graal.

BORGES, L. H., 1999. Sociabilidade, Sofrimento Psíquico e Lesões por Esforços Repetitivos em Processos de Trabalho Repetitivos. Tese de Doutorado, Rio de Janeiro: Instituto de Psiquiatria, Universidade Federal do Rio de Janeiro.

BRASIL, 1997. Portaria no 3.751 de 1990. Altera a redação da Norma Regulamentadora 17 - Ergonomia. In: Segurança e Medicina do Trabalho. Manuais de Legislação Atlas, v. 16, pp. 212-215, 38a Ed., São Paulo: Editora Atlas.

CHATTERJEE, D. S., 1987. Repetition strain injury - A recent review. Journal of Social and Occupational Medicine, 37:100-105.

DEJOURS, C., 1980. Travail: Usure Mentale - Essai de Psychopatologie du Travail. Paris: Centurion.

DUARTE, J. C., 1998. Movimento da Consciência de um Trabalhador com LER - Um Estudo de Caso. Dissertação de Mestrado, São Paulo: Pontifícia Universidade Católica de São Paulo.

GORZ, A., 1987. Adeus ao Proletariado - Para Além do Socialismo. Rio de Janeiro: Editora Forense-Universitária. 
HALL, E. M., 1989. Gender, work control, and stress: a theoretical discussion and an empirical test. International Journal of Health Services, 19:725745.

HIRATA, H. S., 1988. Formação na empresa, educação escolar e socialização familiar: Uma comparação França-Brasil-Japão. Educação e Sociedade, 10:2947.

KURPPA, K., 1979. Peritendinitis and tenosynovitis A review. Scandinavian Journal of Work and Environmental Health, 5:19-24.

LACAZ, F. A. C., 1997. Saúde dos trabalhadores: Cenário e desafios. Cadernos de Saúde Pública, 13:7-19.

LIMA, A. B., 1997. Da Condição de Sujeitado para a de Ator Social: A Construção Social da Militância em um Movimento Social de Portadores de Lesões por Esforços Repetitivos (LER). Dissertação de Mestrado, São Paulo: Pontifícia Universidade Católica de São Paulo.

LIMA, A. B. \& OLIVEIRA, F., 1995. Abordagem psicossocial da LER: Ideologia e culpabilização e grupos de qualidade de vida. In: LER. Diagnóstico, Tratamento e Prevenção. Uma Abordagem Interdisciplinar (W. Codo \& M. C. Almeida, org.), pp. 136-159, Petrópolis: Editora Vozes.

LUCIRE, Y., 1986. Neurosis in the workplace. Medical Journal of Australia, 145:323-327.

MINAYO-GOMEZ, C. \& THEDIM-COSTA, S. M. T., 1997. A construção do campo saúde do trabalhador: Percurso e dilemas. Cadernos de Saúde Pública, 13:21-32.

MPS (Ministério da Previdência Social), 1993. L.E.R.: Lesões por Esforços Repetitivos. Normas Técnicas para Avaliação da Incapacidade. Brasília: MPS.

NAVARRO, V. L., 1999. A Produção de Calçados em Franca (SP): A Reestruturação Produtiva e seus Impactos sobre o Trabalho. São Paulo: Pró-Reitoria de Pesquisa, Universidade de São Paulo. (mimeo.)

OFFE, C., 1989. Capitalismo Desorganizado. São Paulo: Editora Brasiliense.

REGO, M. A. \& SANTANA, V. S., 1995. Associação entre trabalho repetitivo e sintomas psíquicos. In: III Congresso Brasileiro, II Congresso Íbero-americano, I Congresso Latino-americano de Epidemiologia, Resumos Epid95, p.145, Salvador: ABRASCO/ Sociedade Íbero-americana/Associação LatinoAmericana de Medicina Social.

RIBEIRO, H. P., 1995. Estado Atual das Lesões por Esforços Repetitivos (LER) no Banco do Estado de São Paulo. Caderno de Saúde 1. São Paulo: Associação dos Funcionários do Banco do Estado de São Paulo.

RIBEIRO, H. P., 1997. Lesões por esforços repetitivos (LER): Uma doença emblemática. Cadernos de Saúde Pública, 13:85-93.

ROCHA, L. E., 1989. Tenossinovite como Doença do Trabalho no Brasil. A Atuação dos Trabalhadores. Dissertação de Mestrado, São Paulo: Departamento de Medicina Preventiva, Faculdade de Medicina, Universidade de São Paulo.

RODRIGUES, A. M., 1978. Operária, Operário - Estudo Exploratório sobre o Operariado Industrial da Grande São Paulo. São Paulo: Símbolo.

RODRIGUES, A. M., 1992. Lugar e imagem da mulher na indústria. In: Uma Questão de Gênero (A. O. Costa \& C. Bruschini, org.), pp. 266-288, Rio de Ja- neiro: Rosa dos Tempos/Fundação Carlos Chagas. SALERNO, M. S., 1999. O Trabalho na Indústria Contemporânea: Entre Autonomia e Precarização, Inserção e Exclusão. São Paulo: Pró-reitoria de Pesquisa, Universidade de São Paulo. (mimeo.)

SÃO PAULO (Estado), 1992. Norma Técnica sobre Lesões por Esforços Repetitivos. Diário Oficial do Estado de São Paulo, vol. 102 (108), pp. 16-17, 9 jun., seção I.

SATO, L.; ARAÚJO, M. D.; UDIHARA, M. L.; FRANCO, M.; NICOTERA, F. N.; DALDON, M. T.; SETTIMI, M. M. \& SILVESTRE, M. P., 1993. Atividade em grupo com portadores de LER e achados sobre a dimensão psicossocial. Revista Brasileira de Saúde Ocupacional, 79:49-62.

SATO, L., 1992a. Grupo de Qualidade de Vida com Pacientes Portadores de LER. São Paulo: Divisão de Doenças Ocasionadas pelo Meio-ambiente, Centro de Vigilância Epidemiológica, Secretaria de Estado de Saúde de São Paulo. (mimeo.)

SATO, L., 1992b. A LER e a Dimensão Psicológica. São Paulo: Divisão de Doenças Ocasionadas pelo Meio-ambiente, Centro de Vigilância Epidemiológica, Secretaria de Estado de Saúde de São Paulo. (mimeo.)

SATO, L., 1997. Astúcia e Ambigüidade: As Condições Simbólicas para o Replanejamento Negociado do Trabalho no Chão de Fábrica. Tese de Doutorado, São Paulo: Instituto de Psicologia, Universidade de São Paulo.

SETTIMI, M. M.; OLIVEIRA, F.; LIMA, A. B.; SILVESTRE, M. P.; TOLEDO, L. F. \& CAPPUCCI, C. C., 1995. Reinserção de Pacientes de Lesões por Esforços Repetitivos no Mercado de Trabalho: Projeto Piloto - Dados Preliminares da Primeira Fase. São Paulo: Centro de Referência Estadual de Saúde do Trabalhador. Núcleo de Investigação em Trabalho e Saúde/Instituto de Saúde, Secretaria de Estado de Saúde de São Paulo. (mimeo.)

SPINK, M. J. P., 1994. A medicina e o poder de legitimação das construções sociais de igualdade e diferença: Uma reflexão sobre cidadania e gênero. In: A Cidadania em Construção - Uma Reflexão Transdisciplinar (M. J. P. Spink, org.), pp. 105121, São Paulo: Cortez.

SPINK, P., 1992. Saúde mental e trabalho: O bloqueio de uma prática acessível. In: Psicologia e SaúdeRepensando Práticas. (F. C. B. Campos, org.), pp. 91-102, São Paulo: Editora Hucitec.

THOUVENIN, A.; DESCHAMPS, D.; BONNEMĖRE, V.; LATTEUX, M. \& MARIN, M., 1990. Nocivité du geste répétitif forcé. Archives de Maladies Professionelles, 51:251-256.

TRIST, E. L., 1976. Critique of scientific management in terms of socio-technical theory. In: Job Satisfaction (M. Weir, ed.), London: Fontana.

WATZLAWICK, P.; BEAVIN, J. H. \& JACKSON, D. D., 1993. A Pragmática da Comunicação HumanaUm Estudo dos Padrões, Patologias e Paradoxos da Interação. São Paulo: Editora Cultrix. 